\title{
Toyotismo y representación sindical. Dos culturas dentro de la misma contradicción
}

\author{
Battistini, Osvaldo R.*
}

\section{Resumen}

Con la incorporación del capital japonés en el sector automotriz argentino, se utilizan intensamente las técnicas japonesas de organización del trabajo. Este artículo tiene como propósito estudiar las relaciones laborales en una empresa de capitales japoneses en Argentina, con especial atención en la relación entre la implementación del sistema de producción y la acción sindical. Los resultados revelan que factores culturales y políticos impidieron a la empresa contar con una representación sindical en la planta que se adapte fácilmente a sus necesidades, esto fue resistido por el sindicato el cual impuso una representación controlada por un sindicato de rama. Sin embargo la puja por la representación gremial derivó en el abandono de resistencia a imposiciones de la empresa, la cual si bien no pudo imponer un sindicato propio, intentó permear a los representantes obreros con su cultur ra e intereses, quienes al filtrar esta pretensión desarrollaron una actitud reivindicativa propia que se inserta en misma lógica. En definitiva el proceso de construcción de legitimidad para los delegados de planta se inserta en una doble tensión. Por un lado si desarrollan una lógica cercana a la que plantea el sindicato corren el peligro de perder adherentes a los trabajadores, por otro lado, si adoptan patrones de comportamiento fieles a los valores transmitidos por la empresa se distanciarán definitivamente de la asociación gremial perdiendo respaldo y debilitando su capacidad de resistencia con la empresa. Se concluye que en cada formación económico-social la interacción entre los sujetos específicos de la relación capital- trabajo vigente adquiere particularidades que tienen relación con la evolución del proceso cultural y político que permea la relación de fuerzas en la empresa.

Palabras clave: Toyotismo, representación sindical, organización del trabajo, Argentina.

Recibido: 01-08-03 . Aceptado: 01-09-25

Investigador del CONICET. Miembro del CEIL-PIETTE del CONICET. Docente de la Universi dad de Buenos Aires. E-mail: battisti@mail.retina.ar 


\section{Toyota-Ism and Labor Representation. Two Cultures Within the Same Contradiction}

\section{Abstract}

With the incorporation of Japanese capital in the Argentine automobile industry, Japanese work organization techniques are used intensely. The purpose of this article is to study labor relations in a Japanese owned company in Argentina, focusing especially on the relationship between the implementation of the productive system and labor union action. The results reveal that cultural and political factors prevent the company from establishing a labor representation in the plant which easily adapts to its necessities. There was resistance on the part of the labor organization which forced representation under the control of an established automotive labor union. However, the fight over labor representation ended in the abandonment of resistance towards company requirements. The company, although not able to impose its own union, was able to influence work representatives with its culture and interests. The representatives, after understanding this imposition, developed an appropriate labor action within the same logic. The process of constructing legitimacy on the part of labor representatives in the plant was affected by bi-directional stress. If they developed typical labor union logic they would run the risk of losing adherents in the plant, and if they adopted the pattern of faithfulness to values transmitted by the company, they would lose category as a labor association, weakening their capacity of resistance in relation to the company. The conclusion is that in every economic-social formation, interaction between specific players in the capital-labor relation acquires particularities that have relation with the evolution of the cultural-political process that permeates power relations within the company.

Key words: Toyota-ism, union representation, labor organization, Argentina.

\section{Introducción}

La implementación de técnicas japonesas de organización del trabajo en las empresas terminales automotrices se extiende en Argentina desde principios de los años '90. En algunas firmas, especialmente aquellas que ya estaban radicadas en el país, fueron implementándose alternada y paulatinamente solo algunos aspectos derivados de dichas técnicas. Por una parte, la instrumentación de las mismas se vio confrontada con la inexperiencia de los cuadros directivos y técnicos de las empresas, en cargados de su puesta en marcha, con la resistencia cultural de los colectivos de trabajo existente y con la acción sindical para contrarrestar los efectos en las relaciones de trabajo. Por otra parte, la inestabilidad del capital, que se manifestó, durante toda la última década del siglo $X X$, en un proceso dinámico de transferencias y fusiones, incidió en la indeterminación de los lineamientos organizacionales emanados desde las distintas casas matrices $y$, por lo tanto desde los cuadros directivos que se sucedían al frente de las plantas nacionales. En muchas ocasiones, el discurso empresario y su contraste con la 
realidad en las propias firmas reflejaba el desconocimiento de la verdadera esencia del proceso originalmente diseñado en el seno de la producción japonesa.

Por el contrario, la inserción directa del capital japonés en el sector automotriz argentino, a través de Toyota, significó el intento más serio por desarrollar con fidelidad las técnicas organizativas del proceso productivo propias del modelo.

Los directivos de esta nueva empresa gestionaron, antes de su instalación, un modelo particular de relaciones laborales, seleccionaron la mano de obra y la amoldaron a sus necesidades $y$, fundamentalmente, introdujeron una cultura distintiva de trabajo a partir de la concientización de los trabajadores en función de la imbricación de cada uno de los elementos del modelo productivo. Se generó entonces una relación de particulares características frente al sindicato que representaría en adelante a los trabajadores en esa terminal. Ambas partes debieron amoldar aspectos derivados de su propia cultura, pero en ese camino se pusieron de manifiesto contradicciones que afectan principalmente a la estructura gremial, pero que no dejan de incidir en el resultado final del proceso productivo. Además, en ese contexto, la representación sindical en la planta aparece mediada en forma permanente por este juego entre la empresa y la organización gremial.

En el presente trabajo estudiamos la evolución de las relaciones laborales en una empresa de capitales japoneses, poniendo especial atención en la relación entre la implementación del sistema de producción y la acción sindical. Para ello analizamos, en primer lugar, la forma como se desarrolló la inserción de Toyota en Argentina y sus primeros pasos en la relación con el mundo sindical nacional. En segunda instancia describimos las características de la contratación del personal y su evolución frente a las especificidades de la organización de la producción y cultura del trabajo. Luego estudiamos el proceso de trabajo y sus implicancias en la relación con los trabajadores. Dado que la negociación colectiva presenta los principales aspectos que serían desarrollados en las relaciones de trabajo y productivas en la empresa, analizamos en el siguiente apartado los aspectos fundamentales de las negociaciones desarrolladas (convenios y acuerdos) por el sindicato y Toyota. Luego, indagamos acerca de la relación entre la empresa y el sindicato, teniendo en cuenta el papel que, frente a ella, juega la representación en el lugar de trabajo, pero estableciendo referencias permanentes respecto al conjunto de la estructura sindical. Basamos esta última etapa de nuestro trabajo en material derivado de entrevistas en profundidad efectuadas con dirigentes sindicales del Sindicato de Mecánicos y Afines del Transporte Automotor SMATA, con la representación de los trabajadores en la empresa y con directivos del área de recursos humanos de la misma.

\section{Inserción de Toyota en Argentina}

La estrategia de ingreso del capital japonés al país, para la puesta en marcha de esta empresa, se desarrolló a partir del establecimiento de una asociación con la firma argentina Decaroli, que hasta ese momento estaba dedicada sólo a la fabricación de chasis para mi- 
cros de larga distancia, en su planta de la ciudad de Rosario. En la cooperación, ambas empresas contaban con el 50\% de la inversión. Los japoneses aportaron el know-how y la tecnología, mientras que Decaroli incorporó el conocimiento del mercado nacional.

Este proceso comienza en 1994, pero dadas las características especiales del modelo productivo a implementar y de la cultura de Toyota respecto a las relaciones laborales, los empresarios japoneses insistieron acerca de la necesidad de contar con relaciones de trabajo estabilizadas y una representación sindical en la empresa. El desarrollo de la negociación por productividad y los antecedentes convencionales de varias terminales automotrices allanaron el camino para que la primer premisa pudiera cumplirse sin mayores sobresaltos. La segunda condición empresaria encontraría dificultades insalvables en el modelo sindical argentino, por lo cual se debía abandonar la idea de contar con un sindicato propio (tal como sucede en Japón y se pretendía por parte del capital) para negociar con representaciones gremiales a nivel de la actividad o la rama.

El marco estructural que se venía prefigurando en el país y la tendencia flexibilizadora generada por el gobierno hasta ese momento ofrecían perspectivas para el establecimiento de relaciones laborales "seguras" para el sector empresario. De cualquier modo, un adecuado complemento de las mismas sería la fir- ma de un convenio colectivo antes de la puesta en marcha de la planta. Las experiencias de General Motors y Fíat daban la pauta de que esto era posible de realizar dentro del esquema de relaciones de trabajo vigentes. Sin embargo, Toyota iba a establecer una diferencia de substancial importancia respecto a la representación de los trabajadores, ya que serían los empresarios quienes determinarían en última instancia que sindicato tendría a cargo esa función.

La representación de los trabajadores de Decaroli pertenecía a la Unión Obrera Metalúrgica (UOM) y los empresarios argentinos mantenían buenas relaciones con el dirigente de la seccional San Nicolás, Naldo Brunelli, donde la planta estaba ubicada, por lo cual presentaron esa alternativa a sus socios japoneses. Sin embargo, después de un trabajo de exploración y comparación con el SMATA ${ }^{1}$, sobre todo realizada por directivos argentinos del área de recursos humanos, contratados por los japoneses, la elección final recayó en este último gremio.

La decisión estuvo basada en que este último sindicato estaba abierto a la negociación de convenios por empresa, actitud que no presentaba la UOM. Además, en esa misma época, el sindicato de mecánicos había firmado el convenio con la empresa Fíat, después del fracaso de las negociaciones de esta última con los metalúrgicos. Este hecho se constituyó en un argumento muy fuerte a la hora de

1 Los representantes de Toyota mantuvieron entrevistas con sus similares de otras terminales automotrices radicadas en el país, con el objetivo de conocer cual era la relación de cada uno de ellos con el sindicato. 
mostrar a los inversores japoneses las ventajas del SMATA.

Finalmente, con la instalación de la fábrica en Zárate, provincia de Buenos Aires, la inversión japonesa llegó al $100 \%$ y se disolvieron los lazos con Decaroli.

\section{Características del personal}

Antes de la instalación definitiva de la planta se inició un proceso de contratación y entrenamiento de los trabajadores que se desempeñarían en puestos claves de la organización productiva. Un año y medio antes de la puesta en marcha del primer turno de trabajo, en marzo de 1997, se contrató a un grupo de personas con nivel técnico de formación, quienes fueron entrenados durante seis meses en Japón, trabajando como operarios en la línea de producción de la casa matriz. Este personal ocupó luego puestos de conducción en la producción de Toyota Argentina.

En marzo de 1998 se incorporó un segundo turno de trabajo y se promocionó a otros trabajadores a puestos de liderazgo de grupos o de sectores. Producto de esta doble lógica de reclutamiento de este tipo de trabajadores, en la actualidad conviven en la planta dos clases de líderes, aquellos que ingresaron después de su entrenamiento en la casa matriz, desempeñándose directamente en esas tareas y otros que fueron promocionados después de haber realizado tareas directamente productivas en las células. Según representantes de la Gerencia de Recursos Humanos (GRH) de la empresa se plantean diferencias, en la relación con el resto de los trabajadores, entre estos dos grupos. La autoridad de los primeros está más validada que en el caso de los segundos, ya que desarrollaron la tarea de conducción desde el momento en que la fábrica se puso en funcionamiento, en cambio el otro grupo de supervisores tiene ciertas dificultades en establecer con precisión la "línea de autoridad".

El entrenamiento del personal tuvo fundamental importancia para la puesta en marcha del Sistema de Producción Toyota $(\mathrm{SPT})^{2}$, pero previamente se requería una mano de obra preparada para la asimilación de una nueva cultura de trabajo. Todo el personal contratado por la empresa, aún el correspondiente al área de producción, contaba con estudios secundarios completos o finalizaban en el transcurso de ese año esa etapa de su educación formal. En algunos casos estaban comenzando sus estudios universitarios. Se trataba de jóvenes que, en la mayor parte de los casos, no tenían ex-

EI SPT consiste en una red de distribución en el seno de la empresa y entre sus proveedores. Se basa en la idea de T. Ohno de "producir aquello que se haya vendido". El punto de partida es el pedido emanado del cliente, a partir del cual se establece el plan de producción de Toyota y se da todos los días la orden de fabricación de automóviles a la cabeza del taller de carrocerías, desde donde se comienza el sistema de órdenes de producción en toda la planta, así el sistema se desarrolla desde allí hasta el taller de montaje final. Cada una de las autopartes necesaria para el montaje es entregada por los proveedores internos y externos siguiendo las reglas del justo a tiempo (Shimizu, 1999). 
periencia laboral. Los directivos japoneses preferían incorporar trabajadores que no contaran con antecedentes laborales en otras empresas (mucho menos del sector automotriz) con el objetivo de evitar el traslado de "vicios" o costumbres correspondientes a otra lógica productiva. El promedio de edad de los operarios era de 20 años. Según la GRH, la necesidad de generar un proceso de trabajo asentado fuertemente en la calidad llevó a este tipo de reclutamiento de personal.

La capacitación específica estuvo dirigida a lograr la polivalencia de todos los trabajadores, pero fundamentalmente se atendía a la necesidad de lograr una cultura particularmente adaptada a las normas del SPT. Los delegados de la comisión interna sindical de la empresa aseguran que, "se buscaba gente sin experiencia laboral ni cultura sindical, la empresa se encargaba de alimentarlos con la cultura Toyota. Ellos te pulían...".

Estos mismos trabajadores sostienen que lo que se privilegiaba era la edad y no la formación. La primera selección se realizó en los colegios secundarios de Zárate y Campana, luego, al haber agotado las posibilidades de reclutamiento en esas localidades, se recurrió a colegios de otras localidades de la provincia de Buenos Aires, como Baradero y San Pedro. Aproximadamente el $50 \%$ del plantel tuvo en Toyota su primer empleo.

Una vez efectuada la selección y desarrollado el correspondiente entrenamiento se privilegió la realización de contratos por duración indeterminada. El empleo seguro y estable parece ser parte del acuerdo para la aceptación, sin resisten- cia de la lógica productiva. En los momentos de mayor actividad, sólo hay un número pequeño de trabajadores contratados en forma temporaria (entre 15 y 20), mediante agencias de trabajo eventual. Si bien, en el convenio se habilitó el período de prueba a seis meses, sólo se lo utilizó en plazos de 3 meses, pero luego todos los trabajadores luego derivados a contratos por tiempo indeterminado.

\section{El Sistema de Producción Toyota}

El proceso de trabajo está asentado fundamentalmente en las técnicas japonesas de organización. Las distintas secciones se estructuran en base a células de trabajo compuestas por trabajadores polivalentes y cada una de ellas cuenta con un Team Leader. Estos grupos, a su vez, son supervisados por un Foreman, y por sobre ellos existe un Jefe de Sección.

El abastecimiento de materiales se realiza mediante el sistema Kanban, que consiste en el desarrollo de una lógica particular de información y distribución de materiales destinada a controlar de modo armónico las cantidades producidas. Se basa en la utilización de una ficha que es introducida en una funda de plástico y colocada por los miembros de cada uno de los grupos de producción en un dispositivo al costado de su área de trabajo para solicitar a la sección logística la autoparte que el proceso requiere en cada momento del mismo. El abastecimiento, realizado de este modo, hace que no se acumulen piezas a lo largo de la cadena de montaje y sólo se ubiquen 
aquellas que la programación diaria requiere. ${ }^{3}$

La detección de defectos en la fabricación se realiza mediante el autocontrol (Jidoka) de los trabajadores para evitar defectos introducidos por la maquinaria o por el desarrollo de la línea de producción en serie. Los operarios detectan, en forma automática, las anormalidades del proceso. Si ocurre alguna anormalidad en la línea de producción, los trabajadores accionan un mecanismo por el cual se enciende una luz en un tablero ubicado en un lugar visible al team leader. Este cuadro de luces y el sistema de aviso por parte de los trabajadores se denomina Andon. Si un trabajador requiere ayuda por causa de un retraso en su tarea enciende una luz amarilla en el Andon. En caso de tener que parar la línea para resolver algún problema de las máquinas, enciende la luz roja. Además, gran parte de las máquinas automáticas de ensamblado de piezas poseen dispositivos especiales para detectar defectos de fabricación. Este mecanismo es denominado como "a prueba de errores" o poka yoke $e^{4}$ y está destinado a prever los trabajos mal realizados con el objetivo de evitar la propagación de defectos y su acumulación en la producción final. (Monden, 1993)

Para la GRH, el desarrollo del Sistema de Producción Toyota "permite construir la calidad en el mismo proceso de trabajo, a partir de incentivar a los trabajadores para que se involucren con la producción y la calidad del producto..."

La producción se programa en forma mensual, de acuerdo a los pedidos de las concesionarias. Esto impone incluso la introducción de modificaciones permanentes en la misma. Los operarios deben estar preparados para modificar sus tareas de acuerdo al ritmo de la producción $y$ al modelo a fabricar. De cualquier modo, debe tenerse en cuenta que, hasta el momento, las variaciones no son de suma importancia puesto que la planta argentina está dedicada a la fabricación de camionetas Haylux, con lo cual sólo puede tratarse de detalles alternativos a un solo tipo de producto.

A pesar de que se dota a los trabajadores de una formación inicial, de acuerdo a las características fundamentales del SPT, el entrenamiento se realiza en forma permanente, en el puesto de trabajo (job training). Así, se van agregando capacidades a los trabajadores, en forma sucesiva, y en base a la rotación de puestos en la célula, para que vayan adquiriendo, en forma paulatina, todas las habilidades que requiere el proceso productivo. El líder de equipo es el encargado de determinar esta rotación. Este último está capacitado para reemplazar y enseñar el trabajo a cualquier operario. Su tarea, junto a la del supervisor, no está basada

3 Pueden utilizarse Kanban de transporte o de producción. El primero de ellos indica la cantidad de unidades a recoger por el proceso subsiguiente, mientras que un Kanban de producción señala la cantidad a producir en el proceso anterior. Para un mayor detalle de este sistema ver Monden (1993).

4 El poka yoke es un dispositivo que puede inmovilizar la máquina-herramienta cuando una pieza no está correctamente colocada a causa de una distracción de un operador o un defecto de calidad de la misma. (Shimizu, 1999). 
en el control de los miembros de las células (teams members), sino en el apoyo a las actividades de las mismas.

Aunque no se produzca en forma demasiado frecuente, existe la posibilidad que los trabajadores cambien de puesto entre distintas secciones, para lo cual estos deben estar preparados.

En esta planta sólo se realiza el ensamblado final de la camioneta Hailux, en sus distintos modelos. Los componentes son de origen japonés, brasileño y nacional, cuyo stock es relativamente bajo, y depende de las demoras de producción de las autopartistas nacionales y los tiempos requeridos para efectuar las importaciones. En base a estas premisas la planta funciona siguiendo las pautas del modelo de just in time. Esto obligó a Toyota a realizar una transferencia de know-howa los proveedores nacionales para que se adapten a los requerimientos que imponía dicho sistema ${ }^{5}$.

Se le otorga fundamental importancia a la introducción de mejoras en la producción por parte de los trabajadores. Esto se lleva a cabo por dos métodos, el Sistema de Sugerencias y el Mejoramiento Continuo o Kaïzen. En el primero de ellos se incentiva en forma permanente a los operarios a que propongan cambios 0 mejoras en materia de calidad, ergonomía, seguridad, etc. Retribuidos con premios para las mejoras sugeridas e implementadas. Según la GRH este sistema no está orientado directamente a buscar una disminución de costos en la producción. De cualquier modo debemos destacar que la lógica de este instrumento de gestión es el aumento de la eficiencia productiva y en definitiva la reducción de los costos con el consiguiente aumento del beneficio empresarial.

Los grupos Kaïzen, pueden estar constituidos por trabajadores de una misma sección o incorporar trabajadores de más de un sector de la empresa. Las correspondientes reuniones de trabajo se realizan en horas extras y los grupos cuentan con asesoramiento del personal del área de recursos humanos, especialmente entrenados en estas técnicas. Se establece un ranking con todos estos grupos, y se otorgan premios para los ganadores. Según la comisión interna sindical, la mejora continua significa un beneficio para la empresa y puede que en algún caso lo sea también para el trabajador, pero en definitiva "siempre es mejora para la empresa" 6 .

Todo el esquema de producción y de relación entre células de trabajo y los propios trabajadores está asentado en la concepción del vendedor-cliente. Cada grupo de trabajo o trabajador es cliente 0 proveedor de su similar en la línea de producción, por lo cual está obligado a entregar un "producto" de calidad y en los tiempos que se requieren para su "satisfacción". Este modelo, es identificado por los

5 Están tercerizados además los servicios de comedor, la seguridad, la limpieza y el transporte.

6 En este caso, debemos tener en cuenta que en la producción Toyota, la Dirección impone la norma Kaïzen siguiendo la planificación anual de la rentabilización y la reducción del precio de costo. Pero la mas importante de estas actividades está dirigida a la gestión de la eficiencia productiva (productividad) y del costo de la mano de obra (Shimizu, 1995). 
propios trabajadores como la existencia de una "cadena de compromisos". Se ve a este esquema relacional como algo natural y no se considera que el compromiso sea con la empresa "sino con los compañeros". Uno de los propios delegados nos decía al respecto que "Hay un compromiso moral. Vos sabés que si hacés las cosas mal lo perjudicas a él (el compañero)".

\section{La negociación colectiva marca el camino}

Como indicamos más arriba, el convenio colectivo de Toyota fue firmado por la empresa con el SMATA, con anterioridad a la puesta en marcha de la producción. Sin embargo, en su texto ya se prefiguran aspectos esenciales de la misma y que permitirán el desarrollo del SPT, asegurando asimismo una relación particular con el sindicato.

De acuerdo al convenio firmado en mayo de 1996 ( $\left.\mathrm{N}^{\circ} 190\right)$ el SMATA asume sólo la representación de los trabajadores directos de Toyota, quedando excluidos aquellos contratados por las empresas proveedoras especializadas, que no correspondan a las actividades normales y específicas de la terminal.

Se especifican sólo dos categorías de trabajadores: operario polivalente y operario polivalente líder. En ambos casos se indica que deben estar capacitados para realizar indistintamente cualquier tarea de la producción, pero en el caso del líder se agrega la capacidad de asistencia y reemplazo de otros componentes de la célula, de acuerdo a las necesidades que indique la producción, así como la idoneidad para efectuar la capa- citación de los operarios en cada una de las funciones correspondientes a dicho grupo de trabajo. Estos últimos son seleccionados también teniendo en cuenta su nivel de liderazgo y control dentro de la célula, en función de sus aptitudes para orientar y guiar el trabajo a partir de los principios del SPT. El líder aparece así, por un lado como uno más en la célula, pero tiene capacidad de control sobre sus compañeros, al mismo tiempo que, ante la ausencia de alguno de ellos, puede realizar cualquiera de las tareas de la misma forma, sin que la producción se detenga.

La relación entre el número de categorías y asignación ampliada de funciones $y$ tareas encuentra en este convenio un fiel exponente. Sin mencionar distinciones entre grados de autonomía, especializaciones técnicas o saberes productivos, el convenio de Toyota clasifica a los operarios indistintamente bajo una misma categoría abriendo la posibilidad que todos ellos puedan realizar una pluri-actividad.

Dado que en el año 1996, aún se encontraban vigentes, se habilitan las modalidades de contratación promovidas de la ley $\mathrm{N}^{\circ} 24.013$ y $\mathrm{N}^{\circ} 24.465 \mathrm{y}$, al mismo tiempo, se acuerda elevar el porcentaje de trabajadores a ser contratados por esta última modalidad al $20 \%$. Como lo mencionamos anteriormente, el contrato de duración determinada (CDD), tal como sucede con todos los caracterizados como promovidos, no resulta corrientemente utilizado por Toyota. En este sentido, creemos que la empresa trata de asegurarse una forma de reclutamiento que le permita contar en el momento de la contratación con la posibilidad de un filtro (el CDD), que podrá ser utilizado en el pe- 
ríodo de selección y entrenamiento, al cabo del cual se conservarán los más motivados (Coutrot, 1999). En el mismo sentido, podemos decir que se amplía a seis meses el período de prueba establecido por la Ley $\mathrm{N}^{\circ} 24.465$.

Tal como sucede en gran parte de los convenios firmados por el gremio, desde 1991, se acuerda el establecimiento de una cláusula de paz social, por la cual las partes "declaran su firme determinación de realizar los mayores esfuerzos tendientes a que no se interrumpa la continuidad de la producción y a que no se afecten los niveles de empleo, buscando resolver los conflictos que pudieran surgir y que fueran susceptibles de afectar el normal desarrollo de las actividades, mediante la efectiva utilización de todos los recursos de diálogo, negociación y autorregulación".

En la jornada de trabajo se introduce el concepto de "Parada de línea en posición fija", como una forma de asegurar la calidad de los productos. Esto significa que la línea de producción sólo se detendrá una vez que se complete un ciclo completo de trabajo, por lo cual los tiempos de las pausas y almuerzo o cena podrán verse acortados o extendidos en 5 minutos, a fin de lograr el ciclo completo de trabajo.

Se establece la modularización de la jornada en base a un promedio anual de 2.133 horas. Este tipo de módulo permite a la empresa contar con tiempo de trabajo disponible para adecuarlo a sus necesidades productivas.

A partir de la negociación por empresa, las jornadas de trabajo han perdido su uniformidad. Desde la firma del convenio colectivo de General Motors distintos modelos de modularización del tiempo de trabajo han pasado a desarrollarse, dentro de los cuales el de Toyota es un fiel ejemplo. Mediante este mecanismo se redistribuyen los tiempos globales de trabajo a lo largo de un período y se logra disminuir los costos de posibles paradas estacionales de la producción. Pero, además, con el sistema de créditos y débitos de horas se induce a los trabajadores a realizar jornadas más prolongadas que la normal y habitual, sin cobrar las horas extras que les corresponderían de acuerdo a la normativa laboral vigente. Las fluctuaciones de la producción, que se genera como resultado de la adaptación a las exigencias de un mercado cada vez más cambiante, son absorbidas mediante estos mecanismos. Según la letra del convenio las partes llegaron a este tipo de acuerdo con el objetivo de proteger el nivel de empleo. De esta forma se aseguraría la estabilidad de aquellos que ya están contratados en la empresa, pero ante la eventualidad de un aumento coyuntural de la producción el peso del trabajo recae en ellos, con la consiguiente prolongación de la jornada diaria, pero sin ningún efecto sobre el mercado de trabajo externo a la empresa.

En una entrevista con un representante de la GRH se nos advirtió que ese número de horas es teórico y no se cumple en su totalidad. Generalmente hay un cúmulo de horas que no se devuelven por que se vence el plazo previsto.

La jornada modular que se negoció para Toyota 2170 horas resulta inferior al total de horas anuales que se trabajarían si la misma se ajustara a la legislación vigente así como al tiempo de trabajo anual en empleos a tiempo completo para nuestro país, para Filipinas y Colombia, 
pero es superior al resto de los países seleccionados, particularmente también frente al de Japón, como puede verse en la Tabla 1.

La remuneración acordada incluye un porcentaje variable de acuerdo a un sistema de pagos por productividad y calidad, cuyo objeto es el reconocimiento de los mayores esfuerzos de cada célula y los objetivos de productividad y calidad establecidos por la empresa. En el cálculo de dicho porcentaje se incluyen parámetros referidos al nivel de producción, la cantidad de vehículos terminados, la cantidad de horas extras trabajadas y el nú mero de vehículos reparados.

El salario es mensualizado para todos los trabajadores y el monto menor es el del operario polivalente al ingreso (\$520), que aumenta de acuerdo a la antigüedad en los tres primeros años (hasta $\$ 570$ ). Para el caso del operario poliva-

\section{Tabla 1}

Tiempo de trabajo anual en empleos a tiempo completo. Países seleccionados (1997)

\begin{tabular}{ll}
\hline Argentina & 2356 \\
Filipinas & 2342 \\
Colombia & 2189 \\
Corea & 2055 \\
Japón & 2017 \\
México & 1984 \\
Reino Unido & 1952 \\
Estados Unidos & 1904 \\
España & 1807 \\
Francia & 1790 \\
Holanda & 1789 \\
Alemania & 1739 \\
Italia & 1710 \\
\hline
\end{tabular}

Fuente: Contartese (2000). lente líder el sueldo inicial es de $\$ 900$ y aumenta hasta $\$ 1060$ a los tres años. Para todos los trabajadores se acordó un adicional del $10 \%$ por especialización y capacitación. Se abona además un vale mensual para canasta familiar, que también se incrementa con la antigüedad, y representa aproximadamente entre el 19 y el $20 \%$ del sueldo básico para el operario polivalente y entre el 12 y el $16 \%$ para el líder. Debe considerarse también la inclusión de un $10 \%$ por especialización y capacitación.

Desde el comienzo del articulado del CCT se destaca la importancia que las partes acuerden el desarrollo de sus relaciones en función de la "filosofía de trabajo" de Toyota, que se relaciona con el desarrollo de "un concepto moderno de productividad, alta tecnología y calidad final de los productos, mediante un sistema moderno de producción". Se liga a esta conceptualización la implementación de nuevas técnicas de producción y trabajo, pero también la necesidad de encontrar vías armónicas de relacionamiento entre el empleador, los trabajadores y el sindicato, evitando todo tipo de conflicto, buscando mejores niveles de productividad y el máximo desarrollo personal y profesional de los trabajadores. Se compromete, de este modo, a la asociación gremial con los objetivos de productividad de la empresa, así como con la puesta en funcionamiento de una cultura de empresa de especiales características.

Las características del discurso utilizado en el convenio parecen, en gran parte del texto, adaptadas a las premisas seguidas por el empleador para captar mercados, aumentar la producción y en definitiva obtener mayores beneficios. En 
este sentido, se establecen lazos directos entre la adaptabilidad y la polivalencia de los trabajadores con la estructura de la producción, el tipo de producto y la tecnología a utilizar, y aún con la prosperidad en el largo plazo y la satisfacción del cliente. El bienestar de los trabajadores se imbrica directamente con la productividad y el éxito de los negocios para Toyota, cuidando en todo momento de incorporar la idea que ello debe alcanzarse en el marco de "relaciones armoniosas".

Se establece el compromiso del sindicato y la empresa para la búsqueda de mecanismos que aseguren la continuidad laboral en el largo plazo, analizando las situaciones que puedan afectar el nivel de empleo y disponiendo asignaciones alternativas de trabajos fuera del ámbito de la fabricación de vehículos o la capacitación en momentos de baja producción ${ }^{7}$.

La letra del CCT deja claro que el proceso productivo se caracterizará en todo momento por su flexibilidad, por lo cual puede generarse la necesidad poner en marcha procesos de reingeniería dirigidos a producir cambios en las metodologías de trabajo, las formas de organización y los avances tecnológicos. Entonces, la estructuración de puestos, funciones y/o tareas no permanecería inmodificable en el tiempo, sino que podría sufrir transformaciones a partir de dichas necesidades.

La filosofía del trabajo y el SPT incluyen entre sus fundamentos aspectos directamente relacionados con la utilización de la fuerza de trabajo y con la coope- ración de los trabajadores. Así, se hace referencia a la necesidad de establecer mecanismos que tiendan a la mejor utilización del tiempo dentro de las células o equipos, para lo cual deben desarrollarse canales de comunicación permanentes entre sus miembros. Se considera que los componentes de una célula deben cooperar entre sí para resolver los distintos problemas que aparezcan en la producción y conducir a ésta a la reducción permanente de tiempo y el alcance de la productividad. El conocimiento integral del proceso de trabajo, las herramientas, las técnicas y las metodologías implementadas por la empresa, es una condición indispensable para ser parte de las células.

El funcionamiento de estas células está directamente ligado a la eficiencia de la producción, para lo cual se establecen parámetros que tienden a incitar a sus componentes hacia la obtención de objetivos precisos (productividad, calidad, seguridad, presentismo, etc.). La responsabilidad global de las células en estas cuestiones es afirmada en el convenio cuando se sostiene que "los problemas se resuelven a través del esfuerzo de equipo. Cuando un miembro de la célula tiene un problema, se considera que es un problema de la célula".

La participación activa, la circulación de información entre los miembros y la libre iniciativa de estos en sus quehaceres productivos, son propiedades atribuidas al método de trabajo en grupo.

La capacitación resulta un objetivo fundamental de la empresa, ya que cons- 
tituye el fundamento de la inserción de los trabajadores en el proceso productivo, pero además permite que cada uno de ellos adhieran a los principios filosóficos de Toyota. El proceso de trabajo en la planta de Argentina está basado sólo en el montaje de la unidad terminada, la mayor parte de los partes se incorporan externamente al mismo, por lo cual las modificaciones de tareas o funciones no requieren una capacitación intensiva de los trabajadores. Consideramos que el hecho que la polivalencia sea una premisa para la contratación de los trabajadores y su inclusión en las células hace que cada uno de ellos conozca desde un principio la mayor parte de las tareas que se llevan a cabo en la empresa. Sin embargo, el convenio hace referencia a la necesidad de implementar la idea del "entrenamiento en el trabajo". En este sentido, creemos que la intensión de esta premisa es que se asegure la transmisión permanente a los trabajadores de los principios filosóficos de la empresa.

La responsabilización de los trabajadores, en los distintos ámbitos de la empresa, respecto a la calidad presente y futura de los productos, se asienta bajo la implementación del Sistema de Mejora Continua o Kaisen. Teniendo como principio que los trabajadores conocen los procesos y productos de fabricación en su totalidad, se garantiza por medio de este sistema un constante mejoramiento de los tiempos, la calidad, los procedimientos de producción y la productividad.

De acuerdo a los términos del art. 9 de la Ley № 14.250 , se establece una contribución solidaria a favor del SMATA, consistente en dos aportes anuales de dos días de remuneración normal y habitual, a cargo de cada uno de los trabajadores no afiliados, comprendidos en el CCT. En este caso, la empresa actúa como agente de retención y deposita la contribución en una cuenta especial a nombre del sindicato. Es importante destacar, que el sindicato pactó esta cláusula ejerciendo la representación "virtual" de los trabajadores de la empresa, ya que ninguno de ellos aún estaban contratados y menos aún afiliados al gremio.

En un acuerdo salarial posterior al convenio (agosto de 1997), las partes acuerdan una "Contribución empresarial para el cumplimiento y desarrollo de los fines culturales, gremiales y otros del SMATA", que debe ser abonada en forma mensual y depositada en una cuenta especial. El monto pactado es de $\$ 6.000$ en un primer momento y luego se acuerda un aumento a $\$ 7.000$ a partir del 1/1/98.

Toyota es el único caso de una empresa con convenio firmado después del año 1991, con la cual se llega a un acuerdo específico para regular un adicional para el líder de célula y reforzar el sistema de pagos por productividad y calidad.

Durante el año 2000 se negoció la renovación del convenio colectivo ${ }^{8}$, pero

8 Los convenios colectivos firmados por el SMATA con las nuevas empresas, como es el caso de Toyota, son renovados a la fecha del vencimiento. No ocurre lo mismo con los convenios correspondientes a empresas que ya estaban en el país antes de 1991, que se mantienen por ur tractividad. Por otra parte, en las empresas nuevas la actividad negocial informal es inferior a la 
en esta ocasión se produjeron diferencias sustanciales respecto a la negociación anterior. La empresa llevaba ya tres años de funcionamiento en el país, por lo tanto la negociación se desarrolló a partir de la experiencia ya vivida en la producción, con personal en la empresa y con la participación de la comisión interna, que tenía conocimiento fidedigno del desenvolvimiento del proceso de trabajo 9 .

Entre los temas negociados se incluyeron los siguientes:

- Fraccionamiento de la licencia anual ordinaria. Para el personal con una antigüedad entre 6 y 10 años, las vacaciones podrán ser divididas en dos períodos: uno de dos semanas (garantizado entre el 9 de diciembre y el 8 de marzo de cada año) y otro de una semana (que será coincidente con el receso escolar). De cualquier modo esta facultad de la empresa dependerá de la demanda de vehículos.

- Se agregan dos días de licencia con goce de haberes, que podrán ser utilizadas indistintamente para: donación de sangre, examen de enseñanza media o universitaria, mudanza o examen prenupcial.

- Se establece tres tipos de ayuda social: una orden de compra en los casos de casamiento; una canasta de productos infantiles para el caso de nacimiento o adopción; un juego de útiles para los trabajadores con hijos en edad escolar.
- Se estableció que el servicio de transporte será provisto por la empresa.

- La empresa otorgará un subsidio de $\$ 10$ por esposa o concubina y $\$ 10$ por cada hijo de los trabajadores.

- El régimen de categorías se modificó estableciéndose 5 niveles para los operarios y 4 para los líderes de células.

- La asignación remuneratoria vacacional pasará a equivaler a un sueldo básico.

- La contribución solidaria de los trabajadores no afiliados al SMATA pasa a ser de $\$ 20$ mensuales.

\section{Una nueva cultura frente a un sindicalismo tradicional}

Las relaciones de trabajo y sobre todo, las que establece la GRH con respecto a cada uno de los trabajadores están fuertemente marcadas por la cultura organizacional de la empresa. Se establece una interrelación entre la cultura de la empresa y la organización de la producción que se refuerzan mutuamente. Por otra parte, esta misma cultura de producción permea las relaciones con el sindicato.

Los delegados no consideran al involucramiento que se logra a través de este sistema, como algo perjudicial para los trabajadores. Reconocen que la empresa obtiene ventajas económicas con

que se da en las empresas de mayor antigüedad en el país. Los convenios nuevos parecen reflejar la realidad de las empresas de manera más fiel que lo que ocurre con los convenios firmados en la ronda 1988/90 (Battistini, 2001).

9 En el mes de mayo de 2001 el convenio aún no había sido homologado por el Ministerio de Trabajo. 
el desarrollo de estas técnicas, pero esperan obtener a cambio, en la futuras negociaciones de un CCT, un reconocimiento en el salario de los trabajadores.

El hecho de haber negociado un convenio sin trabajadores en la planta es caracterizado por la empresa a partir de que, "...la negociación inicial la hicimos en una fábrica sin gente. Fue todo un desafío porque en realidad SMATA también irrumpió mucho en las premisas que daba Toyota. Es un convenio con toda una parte inicial en la que hay un montón de enunciados, más que nada enunciados de buena voluntad. Y hubo una cuestión de mutua confianza que fue muy positivo".

La caracterización como "enunciados de buena voluntad" puede ser engañosa, ya que debe tenerse en cuenta que las premisas indicadas en el comienzo del convenio se refieren directamente a las " $\mathrm{fi}$ losofía de trabajo" que se aplicarían luego en la producción y estructuran el resto de las pautas incorporadas en el SPT.

Para el sindicato, la llegada de Toyota al país constituyó, por una parte, un desafío, ya que debía enfrentar directamente al modelo de organización de la producción que se desarrollaba sólo en alguno de sus aspectos y generando modelos híbridos en otras empresas. Además, la gestión japonesa planteaba ciertas premisas que condicionaban el ordenamiento de las relaciones laborales en la nueva planta. Por otra parte, también fue un nuevo compromiso con el capital, debido a que las características de las relaciones de trabajo y de la filosofía de la producción que imponían el modelo japonés, que condicionaría su relación con los propios trabajadores de esta empresa.
Otro de los aspectos a tener en cuenta por el SMATA era que un nuevo $\mathrm{CCT}$, en el que se incorporaran las flexibilidades que requería el sistema japonés, podía impulsar al resto de las empresas a querer incorporar en sus propios convenios estas temáticas.

Una vez instalada la planta en Zárate, el sindicato decidió abrir una delegación en esa localidad, y además la construcción de la propia representación sindical dentro de la empresa, derivando en un antiguo delegado de Ford la responsabilidad de hacer de nexo entre el sindicato y la empresa. Los delegados actuales consideran que el sindicato incurrió en un error al tratar de imponer una representación con otra cultura, en este tipo de empresa, ya que los trabajadores no se identificaban con esta persona, dificultándose entonces la actividad gremial. Según la perspectiva de estos delegados "Es distinto tener un representante con la cultura de Ford y Volkswagen que alguien con la visión de acá...".

EI SMATA supervisó el proceso de elección de los delegados, que dada la edad promedio de los trabajadores en la planta, también eran muy jóvenes y sin experiencia sindical. En un primer momento, el sindicato trató de mantener una estrecha relación con la comisión interna gremial $(\mathrm{Cl})$ que se había conformado en la empresa, incluyendo a los nuevos delegados en actividades de formación gremial y manteniendo la asistencia permanente de la delegación en Zárate. Pero con el tiempo, la experiencia que fueron adquiriendo los delegados y sus propias características los hicieron alejarse de las posturas del gremio. Según la empresa los delegados de la $\mathrm{Cl}$, "Fueron forma- 
dos, los llevaron a cursos en Volkswagen y otras fábricas. Con lo que puede ser que esta primer etapa fue un poco más ríspida (respecto a la empresa), porque el modelo que les dieron fue el (impuesto por) SMATA Central, (los llevaron) a las demás plantas automotrices. Con lo cual, al principio, tenían un absoluto alineamiento (con el gremio),...ellos en realidad todavía no habían formado su propia identidad sindical. Venia el (dirigente) de SMATA Central y les decía: ustedes tienen que manejarse de esta forma, y se copiaban. Ahora (después de dos años) ellos empiezan a tener vuelo propio y plantean diferencias con SMATA Central."

La primer comisión interna en la empresa debían estar compuesta por 6 miembros. En una primera elección se eligieron tres delegados, entre los cuales se produjo una primer renuncia a los 15 días. Según la GRH, esta dimisión se generó porque una de las personas elegida "no había entendido qué era ser delegada". Tres meses después se eligió un representante más en otra línea y la $\mathrm{Cl}$ pasó a estar conformada por tres delegados. Esta situación fue valorada por la empresa como positiva y estableció una distinción respecto a otras industrias, tal como lo indica la GRH en el párrafo siguiente: "...debo decir que igual estamos mejor que en el resto de la industria porque nuestro nivel de delegados es bajo... Nosotros podríamos tener cinco y tenemos tres nada más, con lo que, en cantidad de horas de trabajo tengo dos (personas) que podrían ser delegados, que son operarios".

En cambio, para la $\mathrm{Cl}$ el hecho que no se haya podido conformar una comisión interna con el número de representantes que se prevé en la legislación y que se ha- yan producido renuncias a la condición de delegados se debió a presiones ejercidas por la empresa. De cualquier modo, reconocen que los tres que quedaron ya tenían ciertas inclinaciones a realizar esa tarea y fueron quienes incentivaron a otras personas a presentarse en la elección, sin que de ello derive el espíritu propio que debe mover a ejercer esa tarea.

En el primer período de la empresa, los delegados trabajaban en la línea. Tenían dos horas diarias para sus actividades sindicales. Pero con el tiempo, esa actividad se volvió un problema para la producción y fueron separados de ella. Uno de los delegados nos decía que "No estando a full respecto al sistema de producción, para el sistema éramos un lastre".

A diferencia de otras empresas, el local gremial está ubicado por fuera de la zona de producción, su puerta de ingreso y sus ventanas dan al parque que rodea la planta. De esta forma, los delegados no tienen a su vista lo que sucede en la planta y los trabajadores deben salir de ella para acceder a su local. Uno de los objetivos de la $\mathrm{Cl}$ es solicitar a la empresa que habilite una comunicación directa con la planta, para facilitar la presencia y el contacto con los trabajadores.

Según la GRH, los delegados de la $\mathrm{Cl}$ también fueron formados en base a la filosofía de la empresa, por lo cual las negociaciones que se realizan en ese ámbito, en ningún momento ponen en cuestión el SPT. "En realidad ellos ya llevan dos años de mandato, finalmente después de dos años de experiencia, te podría decir que están condicionados pero creo que positivamente, por cómo está organizada la planta, están convencidos que es la mejor forma de trabajar -no di- 
cen no, tendríamos que copiar el modelo de Ford o tendríamos que copiar el modelo de Volkswagen".

La misma $\mathrm{Cl}$ reconoce que la posición del SMATA es resguardar a las demás empresas, para que no adopten un sistema de trabajo similar al de Toyota, dado que en ese caso se reduciría el empleo en estas plantas. La posición de los delegados respecto a este modelo de producción es: "Nosotros, no es que defendamos el SPT, aceptamos el SPT."

La relación entre la empresa y la $\mathrm{Cl}$ tuvo dos etapas. En la primera de ellas, la posición relativamente dura de los delegados generaba algo de fricción, pero, según la GRH, con el tiempo, "...están empezan do a cambiar el discurso y están empezando los propios delegados a decir: nosotros antes que SMATA somos Toyota. Están volviendo a construir la identidad con Toyota. Cuando fueron elegidos delegados, (adquirieron) rápidamente "identidad sindical"; (por otra parte nos distinguen los colores) SMATA es verde y Toyota es rojo. $\mathrm{Y}$ ahora ellos buscan identificarse con Toyota y lo manifiestan diciendo: yo soy primero Toyota, si entro a Toyota tengo trabajo sindical, soy operario de Toyota, estoy orgulloso de ser de Toyota."

En un primer momento, la relación entre los directivos de la empresa y el sindicato central era más corriente y fluida, seguramente como derivación de la firma del convenio y la ausencia de una representación clara en la planta. A medida que la $\mathrm{Cl}$ se fue afianzando en su espacio, se convirtieron en interlocutores de los directivos para las relaciones laborales cotidianas y se espaciaron los contactos con la delegación y el sindicato central. Según la GRH, esto último ocurrió debido a que los delegados de la $\mathrm{Cl}$ "viven el día a día en la planta" y conocen fehacientemente las pautas relacionales de los trabajadores en la producción. Los representantes gremiales en la planta conocen los parámetros culturales establecidos por Toyota, lo cual contrasta con la mirada externa que puede tener un dirigente sindical externo.

El tipo de organización utilizado por Toyota está asentado en una comunicación permanente entre los operarios y los mandos medios. En cierto sentido, esta relación se establece al distenderse el control personal en la producción. Como producto de esta situación, en muchos casos, los trabajadores tienden a canalizar sus demandas por medio de estas estructuras (team leader, supervisores, jefes de sección), prefiriéndolas por sobre los delegados. La empresa genera mecanismos para que las vías de comunicación con los supervisores sean más fluidas, tal como se advierte en el siguiente párrafo: "El tema está en que sigue siendo más eficiente y resume mejor los problemas cuando el operario en vez de ir a la comisión interna va a su supervisor... Ese canal es mucho más aceitado. Parte del sistema de producción de Toyota está organizado así, que el supervisor no sólo resuelve los problemas de producción, sino los personales, de cualquier índole. Entonces, está tan arraigado en la cultura el mando japonés que un supervisor cuando pide algo para un operario es razonable, instantáneamente se resuelve. En cambio cuando viene vía delegados, lamentablemente siempre algún filtro tiene que pasar... La pregunta es: ¿por qué me lo pedís vos (por el delegado) y no me lo pide el operario directamente? Enton- 
ces, a veces termina siendo un poco más largo el camino vía comisión interna que vía supervisor".

Esto puede generar cierto grado de conflicto entre la $\mathrm{Cl}$ y la $\mathrm{GRH}$, ya que se estaría generando competencia entre estos dos niveles, minando la representatividad en la empresa. Según la GRH, se instala entre los trabajadores la idea de que "podría no haber delegados, ya que los problemas se resuelven igual".

El nivel de afiliación en la empresa es relativamente bajo, si se lo compara con otras terminales automotrices. Sólo entre el 30 y el $40 \%$ de los operarios están afiliados al sindicato. Para la $\mathrm{GRH}$, la obra social puede ser el único atractivo para generar la adhesión al gremio, y esto sucede al incrementarse la necesidad de contar con este recurso por parte de los trabajadores, en el momento en que deciden formar una familia. El delegado sindical de la zona cuestiona la posición de los trabajadores de esta empresa, dado que considera la no afiliación como resultado de una actitud individualista de los mismos. La Cl, en cambio parece no asentar su representatividad en la afiliación de los trabajadores al sindicato, ya que dice contar con el apoyo de todos los trabajadores de la planta, aún de los que no adhieren al sindicato.

Para la empresa, de cualquier modo, la representatividad sigue siendo baja, ya que los trabajadores no se acercan al sindicato y no muestran mucha participación en las elecciones que se realizan en la planta. Por otra parte, no surgen demandas de otros trabajadores por ser delegados sindicales.

Sin embargo, en una elección efectuada en el año 2000 el cuerpo de delega- dos se amplió a dos representantes más, lo cual es considerado por la $\mathrm{Cl}$ como un éxito de su tarea en la empresa y una demostración de la legitimidad alcanzada hasta el momento.

Uno de los factores que ha motivado la identificación con la empresa, por parte de los trabajadores, parece ser el hecho de que hasta el momento no se han producido despidos masivos de personal, ni siquiera en períodos en los cuales bajó el nivel de actividad en la planta. La empresa destinó a quienes quedaban sin trabajo en la línea a tareas de capacitación.

La identificación de los trabajadores con la filosofía de la empresa y la baja tasa de afiliación sindical pueden ser factores que coadyuven para que el nivel de conflictividad dentro de la empresa sea muy bajo. El esfuerzo de los directivos está puesto en evitar que los trabajadores se identifiquen con sus colegas, aún con aquellos que ocupan su mismo espacio de trabajo o con el sindicato, para alcanzar una identificación con la empresa en su conjunto (Coutrot, 1999).

En la comparación con otras terminales, la empresa identifica la situación de Toyota como "un paraíso". Las características de los trabajadores (jóvenes, involucrados con la filosofía de la empresa) los distingue de plantas donde la cultura sindical está instalada y se viven las internas política del gremio.

A pesar que el sindicato considera a su relación con Toyota como complicada y de diferentes características que la que mantiene con las otras empresas, no se observan grandes disidencias entre ambas partes. La dirección japonesa de Toyota, finalmente aceptó la representación del SMATA, a costa de relegar sus 
aspiraciones de tener un sindicato propio en la empresa, tal como sucede en Japón. La conducción centralizada, pareció ser hasta hoy, funcional a las pretensiones de la empresa. El representante de la GRH nos decía, que la actitud tomada por José Rodríguez, al encabezar la firma de un acuerdo en el Mercosur, permitió salvar a la industria y esto, "...como (empresa) terminal, lo tengo que valorar. Si yo hago un sindicato por empresa ¿quién lo va a dirigir? Todavía tendría que (hacer) madurar mucho mis delegados, cuando estos delegados eventualmente empiecen a asumir roles fuera de la estructura de Toyota y empiecen a estar en la estructura de SMATA, a ver cómo eso se comporta, recién ahí yo podría tener más elementos para ver si un sindicato por empresa sería mejor".

Por otra parte, de la frase anterior, podemos ver como el propio directivo de la empresa considera a la representación sindical como una propiedad de la empresa (se refiere a ellos como "mis delegados"). Además, aspiraría a contar con un sindicato de empresa cuando pueda hacerlos más funcionales a las necesidades de la misma.

\section{Conclusión}

En cada formación económico-social la interacción entre los sujetos específicos de la relación capital-trabajo vigente adquiere características particulares definiendo en el mismo sentido al proceso productivo. Si bien "los empresarios y directivos toman la iniciativa y tienen mayor capacidad de anticipación en la disposición de las reglas del juego en el proceso de trabajo, no hay que olvidar que gran parte de la historia, en el sentido de cambio y transformación, de la organización del trabajo, no sólo se debe a los cambios de objetivos en torno a la competitividad o la productividad, sino también a la respuesta empresarial a las limitaciones hacia su discrecionalidad impuestas o practicadas por los trabajadores" (Jódar, 1997). En el caso particular de Toyota Argentina, las características de la relación capital-trabajo adquiere especificidades que tienen relación con la evolución del proceso cultural y político que permea la relación de fuerzas en la empresa.

La intención del capital, en el caso de esta empresa, era de contar con una representación sindical en la planta que se adapte fácilmente a sus necesidades, sosteniendo como hipótesis de máxima el desarrollo de un sindicato de empresa con un convenio colectivo propio. Aún con una relación de fuerzas favorable al capital, factores culturales y políticos impidieron la concreción de la primer aspiración empresaria. Las características del sindicalismo argentino resistieron esa posición e impusieron una representación controlada por un sindicato de rama. Sin embargo, la puja por la representación entre los dos gremios que se disputan la adhesión obrera en la rama derivó en el abandono de posiciones que impliquen mayores resistencias a las imposiciones del empresariado sobre las características de las relaciones laborales en la empresa. En ese sentido, entonces el convenio colectivo se constituyó en la herramienta necesaria para el desarrollo de un cultura productiva a la japonesa.

Pero la contradicción cultural que se resolvía a favor del sindicato con el mantenimiento de una representación propia y 
con la adaptación de las relaciones laborales a las necesidades empresarias, a través del convenio colectivo, tendría su correlato en el desarrollo de contradicciones culturales en el seno del sindicato y aún frente a los intereses empresarios en el seno del proceso productivo.

La organización gremial trató desde un primer momento de crear una representación en la planta de propiedades similares a las que ya contaba en empresas ya instaladas en el país. Las características de la mano de obra (jóvenes con niveles de formación relativamente elevados) resistieron a este tipo de imposición sindical y conformaron una representación más acorde a sus propias características. La empresa, por su parte, si no pudo imponer un sindicato propio, intentó permear a los representantes obreros de su cultura e intereses. Los delegados en la empresa filtraron esa pretensión empresaria, adquiriendo un conocimiento pleno de la lógica de funcionamiento del proceso productivo, pero desarrollaron una actitud reinvindicativa propia, que se inserta en esa misma lógica y genera demandas en función del desarrollo del modelo productivo.

Finalmente podemos señalar que el proceso construcción de legitimidad, para los delegados de planta se inserta en una doble tensión. Por un lado, si desarrollan una lógica estratégica cercana a la que plantea el sindicato corren el peligro de perder adherentes entre los trabajadores; pero por otro lado, si adoptan patrones de comportamiento fieles a los valores transmitidos por la empresa se distanciarán definitivamente de la asociación gremial perdiendo el respaldo que ello significa y debilitando entonces su capacidad de resistencia en la discusión cotidiana con los directivos de la empresa. La evolución del complejo entramado entre los determinantes estructurales, políticos y culturales demarcarán los caminos posibles. De las elecciones que se realicen en cada momento dependerá el modelo de relación y de representación que termina constituyéndose.

\section{Bibliografía Citada}

Battistini, Osvaldo R. (2000). "Stratégies syndicales dans le cadre d'une réalité nouvelle dans les entreprises automobiles argentines", ponencia presentada en el Neuvième Rencontre Internatinonal du GERPISA, 7 al 9 de junio.

Contartese, Daniel H. (2000). "Consideraciones sobre el tiempo de trabajo en nuestro País. Su evolución a lo largo del plan de convertibilidad", ponencia presentada en el III Congreso Latinoamericano de Sociología del Trabajo, Buenos Aires - 17 al 20 de Mayo.

Coutrot, Thomas (1999). Critique de l'organización du travail, Ed. La Découverte \& Syros. Paris.

Jódar, Pere (1997). "Más allá de Braverman. EI enfoque del proceso de trabajo y el problema de la reproducción", en Revista Sociología del Trabajo, № 29, Nueva Época, Siglo XXI, Madrid.

Monden, Yasuhiro (1993). El sistema de producción Toyota, Buenos Aires, Ed. Macchi. Buenos Aires.

Shimizu, Koïchi (1999). Le toyotisme, Ed. La Découverte \& Syros.

Shimizu, Koïchi (1995). "Kaizen et gestion du travail chez Toyota Motor et Toyota Motor Kyushu: Un problème dans la trajectoire de Toyota", en Actes du GERPISA № 13. 